\title{
L'expérience de femmes ayant subi une grossesse non désirée au Sénégal : Une étude qualitative [Compte rendu de la rencontre de partage des résultats]
}

\author{
Fatou Mbow \\ Population Council \\ Nafissatou Diop \\ Population Council \\ Babacar Mane \\ Population Council
}

Follow this and additional works at: https://knowledgecommons.popcouncil.org/departments_sbsr-rh

Part of the Demography, Population, and Ecology Commons, Family, Life Course, and Society Commons, International Public Health Commons, Maternal and Child Health Commons, Obstetrics and Gynecology Commons, and the Women's Health Commons How does access to this work benefit you? Let us know!

\section{Recommended Citation}

Mbow, Fatou, Nafissatou Diop, and Babacar Mane. 2016. "L'expérience de femmes ayant subi une grossesse non désirée au Sénégal : Une étude qualitative [Compte rendu de la rencontre de partage des résultats]," STEP UP Rapport de Rencontre. Dakar: Population Council. 

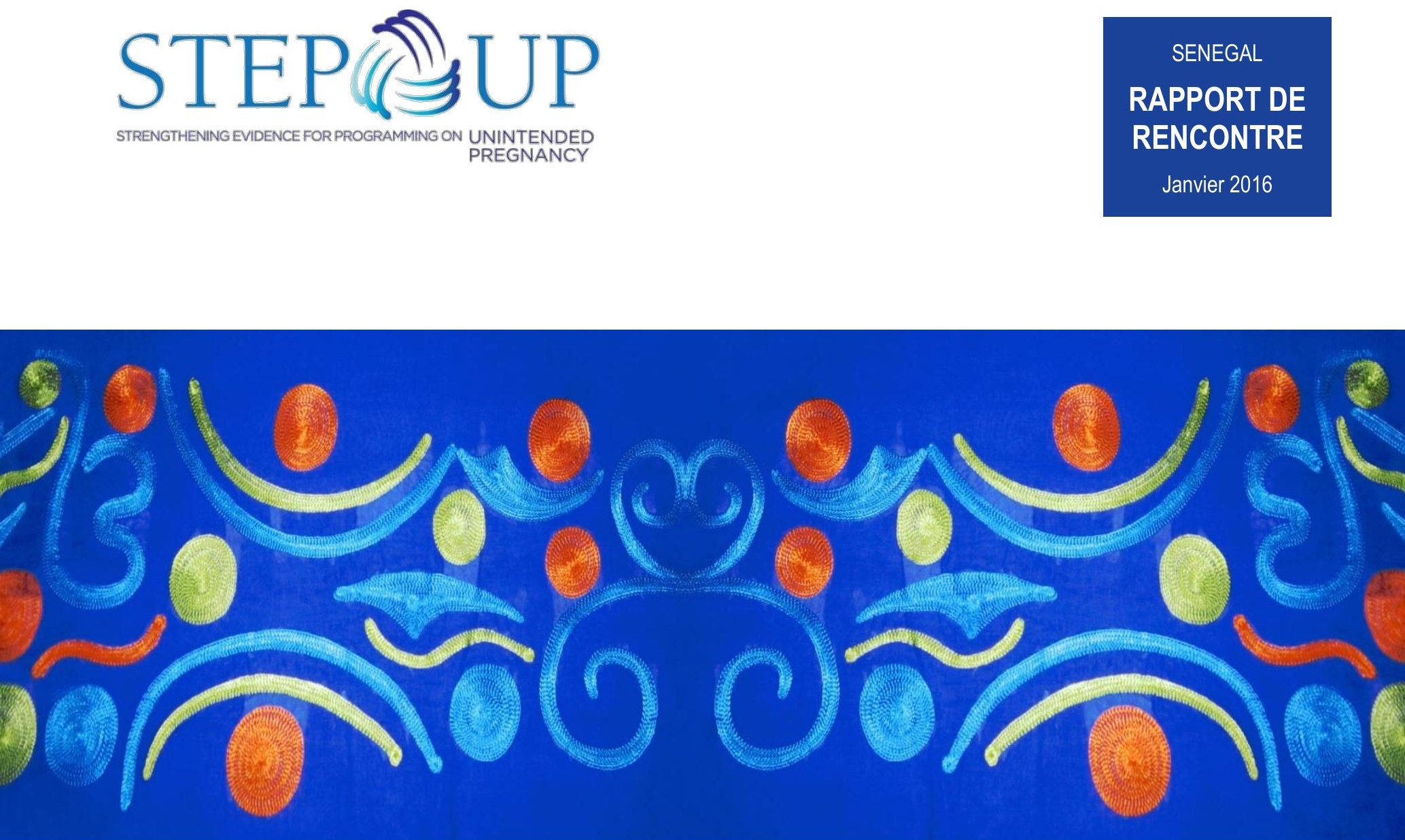

\section{L'expérience de femmes ayant subi une grossesse non désirée au Sénégal: Une étude qualitative}

Compte rendu de la rencontre de partage des résultats

FATOU BINTOU MBOW, NAFISSATOU DIOP, BABACAR MANE

\section{NV




\section{L'expérience de femmes ayant subi une grossesse non désirée au Sénégal : Une étude qualitative}

Compte rendu de la rencontre de partage des résultats

FATOU BINTOU MBOW, NAFISSATOU DIOP, BABACAR MANE

Population Council 
STEP UP œuvre en faveur d'une recherche pertinente à l'élaboration des politiques afin de promouvoir une approche basée sur des données pour améliorer l'accès à la planification familiale et à l'avortement sans risques. Nous travaillons au Bangladesh, dans la partie Nord de I'Inde, au Ghana, au Kenya et au Sénégal. STEP UP est coordonné par le Population Council en partenariat avec African Population and Health Research Center; icddr,b; London School of Hygiene and Tropical Medicine; Marie Stopes International; et Partners in Population and Development. STEP UP est financé par UKaid du Gouvernement de UK. https://stepup.popcouncil.org

\section{POPULATION COUNCIL \\ Ideas. Evidence. Imnact.}

Le Population Council s'attelle aux questions cruciales de la santé et du développement - qu'il s'agisse d'endiguer la progression du $\mathrm{VIH}$, d'améliorer la santé reproductive et d'offrir aux jeunes la promesse d'une vie satisfaisante et productive. Par ses travaux de recherche en biomédecine, en sciences sociales et en santé publique dans 50 pays, il s'efforce d'apporter avec ses partenaires des solutions qui conduisent à des politiques, des programmes et des technologies plus efficaces, pour améliorer la vie partout dans le monde. Fondé en 1952, le Population Council est une organisation non gouvernementale à but non lucratif. II siège à New York sous la conduite d'un conseil d'administration international. www.popcouncil.org

Citation recommandée: Fatou Bintou Mbow, Nafissatou Diop, Babacar Mané. 2016. "L'expérience de femmes ayant subi une grossesse non désirée au Sénégal: Une étude qualitative. Compte-rendu de la rencontre de partage des résultats auprès" Rapport de Rencontre. STEP UP. Dakar: Population Council.

\section{(C) 2016 Population Council}

Pour plus d'informations, veuillez contacter:

Dr Harriet Birungi, hbirungi@popcouncil.org

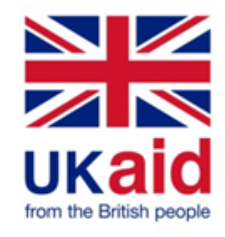




\section{Table des Matières}

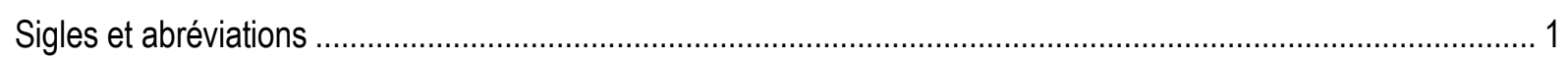

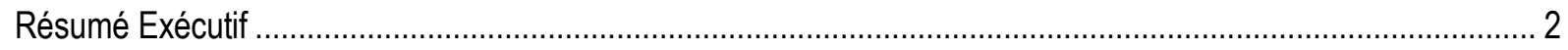

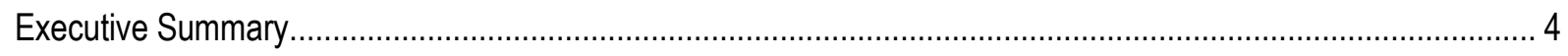

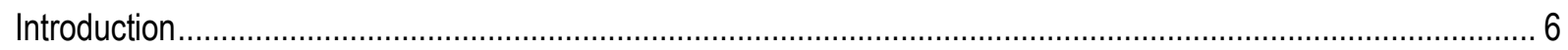

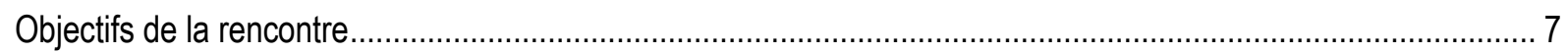

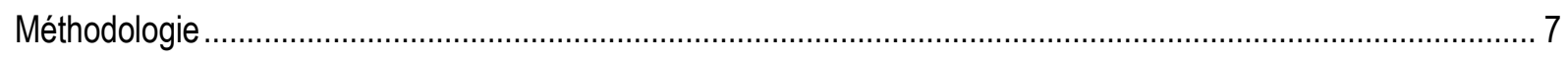

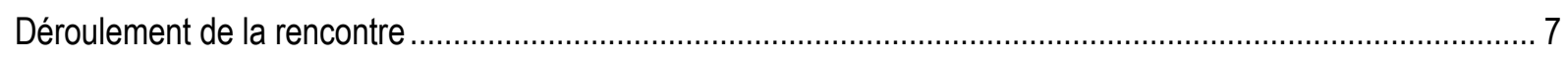

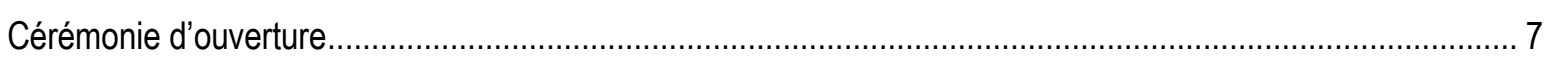

Présentation du contexte, objectifs et méthodologie de l'étude .............................................................. 8

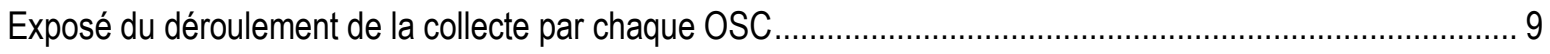

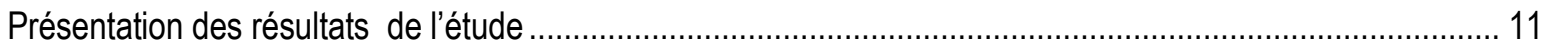

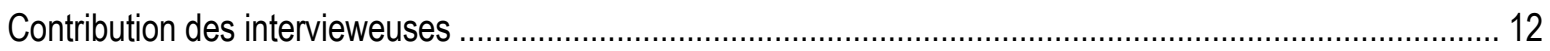

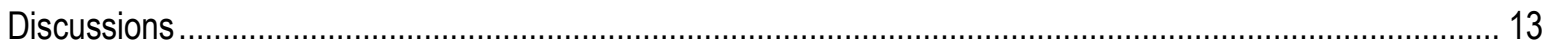

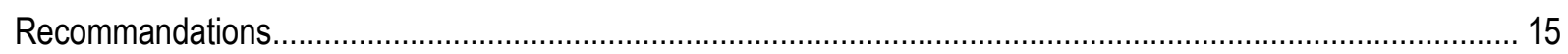

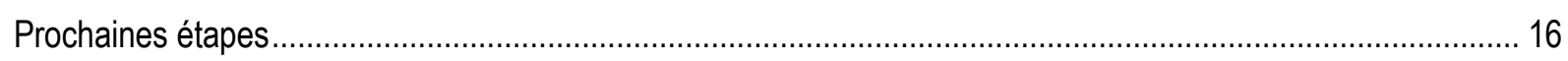

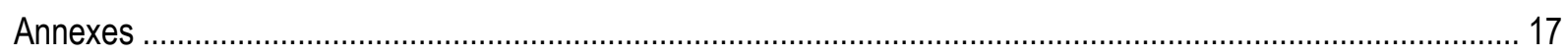

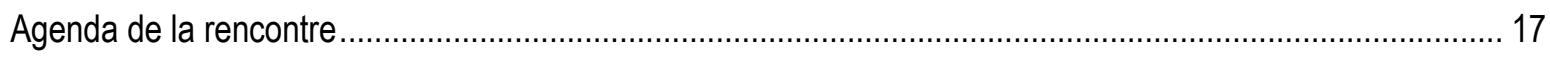

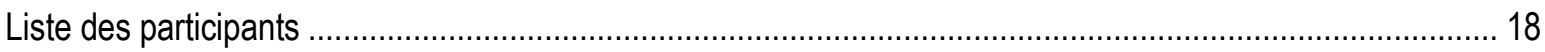




\section{Sigles et abréviations}

AC Animatrices Communautaires

AFEMS Association des Femmes Médecins du Sénégal

AJS Association des Juristes Sénégalaises

ASGO Association Sénégalaise des Gynécologues/Obstétriciens

CNERS Comité National d'Ethique pour la Recherche en Santé

DFID Department for International Development

DPRS Direction de la Planification, de la Recherche et des Statistiques

DSRSE Direction de la Santé de la Reproduction et de la Survie de l'Enfant

EA Entretien Approfondi

GD Groupe de Discussion

MSAS Ministère de la Santé et de l'Action Sociale

OSC Organisation de la Société Civile

OMS Organisation Mondiale de la Santé

PC Population Council

PF Planification Familiale

RSJ Réseau Siggil Jigeen

SR Santé de la Reproduction

STEP UP Strengthening Evidence for Programming on Unintended Pregnancy

TMM Taux élevé de Mortalité maternelle 


\section{Résumé Exécutif}

Au Sénégal, en 2010, environ 3.6 pour cent des décès maternels ont été liés à un avortement à risque, et en 2012, 51500 avortements provoqués ont été reporté. Pourtant, le Sénégal a ratifié plusieurs traités et conventions au niveau régional et international relatifs à la santé de la femme. Malgré cela, le pays a l'une des lois les plus restrictives du monde sur l'avortement -- y compris pour les victimes de grossesse non désirée suite à un viol ou inceste. En 2013, le Comité de plaidoyer pour l'accès à l'avortement médicalisé au Sénégal (aussi appelé la Taskforce) a été créé pour mener des activités de plaidoyer pour la révision de cette loi. Depuis, l'avortement est plus ouvertement discuté. Cependant, la voix des femmes victimes de grossesse non désirée n'est guère entendue.

Dans le cadre du Projet Strengthening Evidence for Programming on Unintended Pregnancy (STEP UP), et sur financement de DFID, une étude qualitative a été menée dans la région de Dakar par le Population Council en partenariat avec la Taskforce sur l'expérience des femmes ayant subi une grossesse non désirée suite à un viol ou un inceste pour apporter la voix de ces femmes au débat sur la légalisation de l'avortement médicalisée.

Suite à l'étude, une réunion de la Taskforce a été organisée pour discuter des résultats et élaborer des recommandations. Une rencontre plus large, dont ce rapport est la synthèse, a été organisée le 20 janvier 2016 à l'hôtel Radisson Blu par la Direction de la Santé de la Reproduction et de la Survie de l'Enfant (DSRSE) et le Population Council pour une dissémination des résultats au niveau national.

Cette rencontre s'est déroulée en plusieurs étapes : elle a débuté par une cérémonie d'ouverture au cours de laquelle la Directrice du Population Council et le Directeur de la DSRSE ont prononcé chacun un discours. Ensuite, la Directrice du Population Council a présenté le contexte dans lequel l'étude s'est déroulée, ses objectifs, et l'approche méthodologique qui a consisté en des entretiens approfondis (EA) réalisés en collaboration avec trois (3) Organisations de la Société Civile (OSC) membres de la Taskforce, à savoir :

Le Réseau Siggil Jigeen (RSJ) qui a interviewé les femmes qui ont subi une grossesse non désirée résultant d'un viol ou d'un inceste, ont gardé la grossesse, et l'enfant est né vivant et a survécu au moins un an (Groupe 1);

- L'Association des Juristes Sénégalaises (AJS) dont le focus était les femmes qui ont subi une grossesse non désirée résultant d'un viol ou d'un inceste, ont tenté d'interrompre la grossesse et ont été sanctionnées par la loi (Groupe 2) ; et

- L'Association des Femmes Médecins du Sénégal (AFEMS) qui a interviewé avec les femmes qui ont subi une grossesse non désirée résultant d'un viol ou d'un inceste et ont souffert de conséquences dramatiques sur leur santé (Groupe 3).

Pour chaque OSC, la coordonnatrice de l'étude a rapporté le déroulement de la collecte pour son groupe. Chacune a insisté sur la durée de la collecte, les procédures de recrutement des participantes, le bilan de leur collecte, ainsi que les difficultés rencontrées.

La Chargée de Programmes du Population Council a ensuite présenté les résultats de l'étude, en commençant par le bilan global de la collecte, précisant qu'au total, sur 36 candidates potentielles identifiées, $15 \mathrm{EA}$ ont été complétés. Elle a aussi décrit les caractéristiques sociodémographiques des femmes interviewées. La Chargée de Programmes a ensuite parlé de l'expérience des femmes par rapport au viol/inceste, et des différentes expériences de la grossesse et ses conséquences. La Chargée de Programmes a aussi souligné que très peu d'actions étaient entreprises par les victimes ou leurs familles contre les agresseurs. En ce qui concerne la loi sur l'avortement, elle a indiqué que les victimes la jugent injuste, avec de nombreuses conséquences (pour les femmes) et qu'elles souhaitent qu'elle soit modifiée. Puis, la présentation a mis en exergue que les femmes 
étaient victimes de stigmatisation et de discrimination, quel que soit leur groupe. Enfin, la présentatrice a expliqué les difficultés liées à ce genre d'étude, avant de conclure et d'exposer les recommandations.

Suite à la présentation, les contributions des intervieweuses ont surtout porté sur la gestion des aspects émotionnels lors des entretiens et la difficulté à garder certaines victimes canalisées sur les objectifs de l'étude.

Les points abordés lors des discussions ont essentiellement eu trait à la pertinence de l'étude car elle constitue un argumentaire solide dans le cadre du plaidoyer pour l'assouplissement de la loi sur l'avortement, à des questions sur le nombre important de refus de participer, aux critères d'éligibilité, à la prise en charge (psychologique, juridique et médicale) des victimes, et à la formation des intervieweuses au vu du rapport du nombre de candidates potentielles et d'interviews complétés. Enfin, des recommandations ont été faites par les participants, avant que les prochaines étapes soient expliquées. 


\section{Executive Summary}

In Senegal, in 2010, approximately 3.6 percent of maternal deaths were related to unsafe abortion, and in 2012, 51,500 induced abortions were reported. However, while Senegal has ratified several treaties and conventions at regional and international levels related to women's health, the country has some of the most restrictive abortion laws in the world-- including for victims of unwanted pregnancy resulting from rape or incest. In 2013, the advocacy committee for access to safe abortion in Senegal (aka the Taskforce) was created to conduct advocacy activities for the revision of those laws. Since then, abortion is more openly discussed. Nevertheless, the voices of female victims of unwanted pregnancy remain unheard.

Through the Strengthening Evidence for Programming on Unintended Pregnancy (STEP UP) Project and with funding from DFID, a qualitative study was conducted in the Dakar region by the Population Council in partnership with the Taskforce on the experience of women who suffered an unwanted pregnancy resulting from rape or incest to bring the voice of these women to the debate on the legalization of safe abortion.

Following the study, a meeting of the Taskforce was organized to discuss the results and develop recommendations. Subsequently, a broader meeting, of which this report is the synthesis, was held on January 20, 2016 at the Radisson Blu Hotel by the Directorate of Reproductive Health and Child Survival (DSRSE) and the Population Council for a dissemination of the results at the national level.

This meeting took place in several phases: it began with an opening ceremony during which the Director of the Population Council and the Director of the DSRSE each delivered a speech. Then, the Director of the Population Council presented the context in which the study was conducted, its objectives, and the methodological approach which consisted of in-depth interviews (IDIs) conducted in collaboration with three (3) Civil Society Organizations (CSO) members of the Taskforce, namely:

- The Siggil Jigeen Network (RSJ) which interviewed women who have had an unwanted pregnancy resulting from rape or incest, kept the pregnancy, and the child was born alive and survived at least one year (Group 1);

- The Association of Senegalese Women lawyers (AJS) whose focus was on women who have had an unwanted pregnancy resulting from rape or incest, attempted to eliminate the pregnancy and were penalized by law (Group 2); and

- The Association of Women Medical Doctors of Senegal (AFEMS) which interviewed women who have had an unwanted pregnancy resulting from rape or incest and suffered dramatic consequences on their health (Group 3).

For each CSO, the study coordinator reported on their group's data collection process. Each emphasized the duration of the data collection, participant recruitment procedures, their data collection result, and the difficulties they faced.

Then, the Population Council Program Officer presented the study results, starting with the overall data collection returns, stating that, out of a total of 36 potential candidates identified, 15 IDIs were completed. She also described the sociodemographic characteristics of women interviewed. The Program Officer then talked about the experience of women subjected to rape/incest, and their various experiences with pregnancy and its consequences. The Program Officer also noted that very few actions were taken by the victims or their families against the perpetrators. Regarding the law on abortion, she indicated that victims find it unfair, with many consequences (to the women) and that they want it changed. Then, the presentation highlighted that women were victims of stigma and discrimination, regardless of their group. Finally, the presenter explained the difficulties associated with this kind of study before concluding and stating the recommendations. 
Following the presentation, the interviewers gave input which focused on the management of the emotional aspects of the interviews and the difficulty of keeping some victims focused the objectives of the study.

Points raised during discussions were mainly related to: the relevance of the study (as it provides strong arguments for the advocacy of the relaxation of the abortion laws); questions about the large number of refusals to participate; the eligibility criteria, support (psychological, legal and medical) for victims; and the interviewers' training considering the number of potential candidates vs completed interviews. Finally, recommendations were made by the participants, before the next steps were explained. 


\section{Introduction}

Au Sénégal, des études relatives aux grossesses non désirées ont fait état de statistiques alarmantes. Elles montrent en effet qu'en 2010, environ 3.6 pour cent des décès maternels étaient liés aux avortements à risque (Ipas-MSAS, 2010), et qu'en 2012, il y aurait eu 51500 avortements provoqués au Sénégal, dont 38 pour cent effectués par des prestataires traditionnels ou non qualifiés (Guttmacher, 2015). Par ailleurs, pendant le 1er semestre 2013, 40 détentions provisoires ont été ordonnées pour infanticide ou avortement provoqué (Direction de l'administration pénitentiaire).

Le Sénégal a ratifié des traités et conventions au niveau régional et international relatifs à la santé de la femme. Malgré cela, ce pays a l'une des lois les plus restrictives du monde sur l'avortement. En 2013, sous l'égide du Ministère de la Santé et de l'Action Sociale (MSAS), un Comité de plaidoyer pour l'accès à l'avortement médicalisé au Sénégal (aussi appelé Taskforce) a été créé pour mener des activités de plaidoyer pour une révision de la loi sur l'avortement. Depuis, l'avortement est plus ouvertement discuté et débattu. Cependant, la voix des femmes victimes de grossesse non désirée n'est guère entendue.

Dans le cadre du Projet Step Up (Consolider les données pour une programmation sur les grossesses non désirées), et sur financement de DFID, une étude qualitative a été menée dans la région de Dakar par le Population Council en partenariat avec la Taskforce sur l'expérience des femmes ayant subi une grossesse non désirée suite à un viol ou un inceste. L'étude permet d'apporter la voix de ces femmes au débat sur la légalisation de l'avortement médicalisée. Elle a pour objectif de développer une meilleure compréhension de la vie des femmes qui ont subi une grossesse non désirée suite à un viol ou un inceste, en particulier en :

- Documentant la vie de ces femmes.

- Aidant à développer des arguments et des messages de plaidoyer pour un assouplissement de la loi sur l'avortement.

- Fournissant des évidences basées sur la recherche à la Taskforce et à tous ceux qui sont intéressés par le sujet pour servir dans les activités de plaidoyer pour un assouplissement de la loi sur l'avortement.

L'étude a consisté en des entretiens approfondis (EA) auprès de trois (3) groupes de femmes :

- Femmes qui ont subi une grossesse non désirée résultant d'un viol ou d'un inceste, ont gardé la grossesse, et l'enfant est né vivant et a survécu au moins un an (Groupe 1).

- Femmes qui ont subi une grossesse non désirée résultant d'un viol ou d'un inceste, ont tenté d'interrompre la grossesse et ont été sanctionnées par la loi. Les femmes pouvaient, au moment de l'étude, être incarcérées, ou avoir purgé leur peine (Groupe 2).

- Femmes qui ont subi une grossesse non désirée résultant d'un viol ou d'un inceste et ayant souffert de conséquences dramatiques sur leur santé. II s'agissait d'interviewer pour ce groupe des victimes éligibles ou des familles de victimes décédées (Groupe 3).

La collecte des données a été réalisée en collaboration avec trois (3) Organisations de la Société Civile (OSC) membres de la Taskforce : le Réseau Siggil Jigeen (RSJ) qui a travaillé avec Groupe 1; l'Association des Juristes Sénégalaises (AJS) pour le Groupe 2 ; et l'Association des Femmes Médecins du Sénégal (AFEMS) pour le Groupe 3.

Suite à l'étude, une réunion de la Taskforce a été organisée pour discuter des résultats et élaborer les recommandations. Le 20 janvier 2016, la Direction de la Santé de la Reproduction et de la Survie de l'Enfant (DSRSE) et le Population Council ont organisé une rencontre de partage à l'hôtel Radisson Blu pour une dissémination plus large des résultats de l'étude au niveau national. 


\section{Objectifs de la rencontre}

La rencontre avait pour objectif de partager les résultats de l'étude avec les participants et de recueillir leurs feedbacks et recommandations en vue de la finalisation du rapport.

\section{Méthodologie}

La rencontre s'est déroulée de manière participative en plénière en plusieurs étapes :

- Cérémonie d'ouverture

- Présentation du contexte, objectifs et méthodologie de l'étude

- Exposé du déroulement de la collecte pour chaque OSC

- Présentation des résultats de l'étude

- Contribution des intervieweuses

- Discussions

- Prochaines étapes

\section{Déroulement de la rencontre}

\section{Cérémonie d'ouverture}

La cérémonie a été présidée par le Directeur de la Santé de la Reproduction et de la Survie de l'Enfant et la Directrice du Population Council. Deux discours ont été prononcés:

- Population Council : la Directrice Pays, a commencé par remercier le MSAS pour la collaboration et le soutien. Ensuite elle a rappelé le taux élevé de mortalité maternelle au Sénégal et qui s'explique en partie par les avortements clandestins dus aux grossesses non désirées, dont les viols et incestes constituent une part non négligeable. Elle a également souligné que l'avortement à risque constituait un problème majeur de santé publique, et que les activités de plaidoyer menées depuis 2013 par la Taskforce pour une révision de la loi sur l'avortement ont contribué à rendre le sujet moins tabou et à permettre de le débattre plus ouvertement en public. La Directrice a souligné que la présente étude sur l'expérience des femmes ayant subi une grossesse non désirée au Sénégal, menée en partenariat avec la Taskforce, s'inscrit dans le cadre du mandat du Population Council de mettre à disposition des données probantes, de haute qualité scientifique, pour une prise de décisions basées sur les évidences, en apportant au débat national la voix de cette cible clé, car directement concernée. Elle a partagé les espoirs du Population Council de voir les résultats de l'étude contribuer à renforcer l'argumentaire pour le plaidoyer pour l'avortement médicalisé et amener le Sénégal à harmoniser ses textes juridiques et législatifs avec ses engagements internationaux, tel que l'article 14 du Protocole de Maputo et l'article 12 de la Convention sur l'élimination de toutes les formes de discrimination à l'égard des femmes (CEDEF). Elle a terminé en remerciant la Taskforce pour la collaboration, et le Projet Step Up et DFID pour le financement de l'étude, et en renouvelant l'engagement du Population Council aux côtés du Gouvernement sénégalais et de ses partenaires, en particulier la DSRSE. 
DSRSE : le Directeur, représentant le MSAS, a commencé son allocution en rappelant les statistiques de 2014 qui montrent qu'au Sénégal environ 31 pour cent des grossesses n'étaient pas planifiées et que ces grossesses étaient souvent le résultat de besoins non satisfaits (BNS) en planification familiale (PF). Des réponses adéquates à ces besoins pourraient contribuer à en réduire de façon significative les conséquences néfastes, parfois dramatiques, y compris infanticides et avortements provoqués. II a souligné par ailleurs la volonté du ministère de faire de la prévention des grossesses non désirées une priorité, cela permettant aussi d'éviter le recours à l'avortement. Le Directeur a signalé l'adoption par le Sénégal au cours de ces dernières années de plusieurs lois et politiques, et la mise en œuvre de programmes directement liés à la santé de la reproduction (SR), y compris à la PF et aux soins après avortement. Selon lui, cela témoigne d'une réelle volonté politique de traiter ces questions, d'autant que les plus hautes autorités du pays se sont récemment déclarées en faveur de l'avortement médicalisé dans certains cas. Tout de même, il fait remarquer qu'en dépit des efforts de plus en plus importants consentis par l'Etat pour améliorer la SR des populations sénégalaises, de nombreux obstacles, notamment juridiques, continuent à freiner les progrès réalisés pour réduire les grossesses non désirées. C'est pour cela, que pour fédérer les actions dans ce domaine, un cadre juridique cohérent doit être mis en place dans les meilleurs délais en vue d'harmoniser les textes au niveau national, et aussi assurer la conformité avec les engagements pris par le Sénégal au niveau international. II a reconnu que le sujet est sensible, mais que la réalité du pays exige que s'ouvre un débat lucide et constructif, tenant compte du contexte socio-culturel et religieux. Le Directeur a terminé en renouvelant l'engagement du Ministère de la Santé à faire avancer la question, qui fait partie intégrante des droits à la santé reproductive des femmes sénégalaises. II a remercié également la Taskforce qui mène depuis sa création en 2013, sous l'égide du MSAS, des activités de plaidoyer pour une révision de la loi sur l'avortement et pour l'accès à l'avortement médicalisé, ainsi que les partenaires techniques et financiers, notamment le Population Council, dont le soutien demeure essentiel pour l'atteinte des objectifs du pays.

\section{Présentation du contexte, objectifs et méthodologie de l'étude}

La Directrice du Population Council, a rappelé le contexte, à savoir :

- L'existence de statistiques indiquant qu'en 2010, environ 3.6 pour cent des décès maternels aurait été liés aux avortements à risque, et qu'en 2012, il y aurait eu 51500 avortements provoqués au Sénégal, dont 38 pour cent effectués par un prestataire traditionnel ou non qualifié.

- La ratification au niveau régional et international par le Sénégal de traités et conventions relatifs à la sante de la femme.

- L'existence au Sénégal d'une des lois les plus restrictives du monde sur l'avortement.

- Les activités de plaidoyer menées par la Taskforce pour une révision de la loi sur l'avortement et qui ont beaucoup contribué à ce que l'avortement soit plus ouvertement discuté et débattu aujourd'hui. La Directrice a cependant reconnu que la voix des victimes n'est pas souvent entendue dans ces débats.

Concernant les objectifs de l'étude, la Directrice a indiqué qu'il s'agissait de développer une meilleure compréhension de la vie des femmes qui ont subi une grossesse non désirée suite à un viol ou un inceste, et plus spécifiquement de documenter la vie de ces femmes, d'aider à développer des arguments et des messages de plaidoyer, et de fournir des évidences basées sur la recherche à la Taskforce et à tous ceux qui sont intéressés par le sujet pour servir dans les activités de plaidoyer pour un assouplissement de la loi sur l'avortement. 
Quant à la méthodologie, la présentatrice a mis l'accent sur :

- Les approbations éthiques (Population Council et Comité National d'Ethique pour la Recherche en Santé (CNERS) du MSAS) et autorisation administrative de la Direction de la Planification, de la Recherche et des Statistiques (DPRS) du MSAS.

- Le partenariat noué avec les 3 OSC, membres de la Taskforce : l'Association des Femmes Médecins du Sénégal (AFEMS), l'Association des Juristes Sénégalaises (AJS), le Réseau Siggil Jigeen (RSJ) pour la collecte des données pour les groupes de victimes.

- Les précautions prises du fait de la sensibilité du sujet, notamment :

- La préparation d'une liste de contacts utiles (professionnels de santé, organisations spécialisées) à fournir aux participantes qui désiraient recourir, à leur frais, à une aide psychologique, juridique ou médicale selon leur besoin.

- La possibilité de mener jusqu'à 3 sessions d'interview avec une participante pour respecter la possible émotivité de se remémorer des évènements douloureux.

- Le fait qu'il s'agisse d'une étude qualitative menée dans la région de Dakar à travers des entretiens individuels approfondis (EA) avec des femmes âgées de 18 ans ou plus au moment de l'interview, mais qui auraient pu avoir été victimes même en étant mineures.

- Le financement de l'étude par DFID, à travers le Programme de Recherche du Consortium «Strengthening Evidence for Programming on Unintended Pregnancies (Step Up) ».

- Le support partiel aux activités de la Taskforce par le Population Council à travers un financement de la Fondation Hewlett.

\section{Exposé du déroulement de la collecte par chaque OSC}

La coordonnatrice de l'étude au sein de chaque OSC a fait un rapport du déroulement de la collecte pour son groupe. Chacune a insisté sur la durée de la collecte, les procédés de recrutement des participantes, le bilan de la collecte pour leur groupe, ainsi que les difficultés rencontrées.

\section{Groupe 1 (RSJ) : Grossesse gardée}

La collecte s'est déroulée du 15 Juin au 30 Août 2015 et s'est déroulée en plusieurs étapes :

- Identification des candidates potentielles avec l'implication de leurs Animatrices Communautaires (AC) aidées par des délégués de quartier et autres collaborateurs de RSJ, collaboration avec des structures telles que la Maison Rose, le Centre Guindi, et le CEGID.

- Vérification par la coordinatrice des critères d'éligibilité.

- Contact de la participante potentielle par L'AC pour lui expliquer l'étude et lui demander si elle est intéressée à participer. Si la femme accepte de participer :

- L'AC informe la coordinatrice qui organise une rencontre entre une intervieweuse formée et la femme.

- L'intervieweuse commence par expliquer de nouveau l'étude à la femme et demande son consentement éclairé.

- L'entretien n'est fait que si la femme donne son accord et signe le formulaire de consentement éclairé. 
- Difficultés rencontrées

- Inflexibilité des critères de recrutement.

- Désistement des victimes à témoigner.

\section{Groupe 2 (AJS) : Avortement / infanticide}

La collecte s'est déroulée du 03 Juin au 30 Août 2015 suivant une méthodologie légèrement différente suivant la catégorie de femmes :

- Pour les femmes détenues:

- Lettre officielle de l'AJS à la directrice de la Maison d'Arrêt pour Femmes de Liberté VI, puis contact par la coordonnatrice pour introduire l'étude et demander la collaboration dans l'identification des candidates éligibles.

- Triage des fiches des détenues avec l'aide de la greffière et

Rencontre par la coordinatrice et le Population Council avec les candidates potentielles pour vérifier les critères d'éligibilité.

- Explication de l'étude à celles qui étaient éligibles, et demande de participation.

- Pour celles qui acceptaient de participer, rencontres avec les intervieweuses formées dans une salle mise à disposition par la Direction de la prison.

- Pour les femmes sorties de prison :

- Message à tous les membres de l'AJS, aux avocats partenaires ou membres de l'AJS qui ont eu à défendre de tels cas, ainsi qu'à l'ONG Tostan.

- Collaboration avec une ancienne détenue.

- En présence d'une femme consentante, l'intervieweuse commence par expliquer de nouveau l'étude et demander le consentement éclairé.

- Difficultés rencontrées

- Refus de participer (notamment les ex-détenues).

- Amalgame entre les objectifs de l'étude et les implications (jugées potentiellement favorables) sur le dossier judiciaire (détenues).

- Manque de confiance.

- Pas de cas d'avortement recrutés. Seuls des cas d'infanticide sont documentés dans l'étude.

\section{Groupe 3 (AFEMS) : Conséquences sanitaires graves}

- La collecte s'est déroulée du 13 Juillet au 30 Août 2015 et s'est déroulée en plusieurs étapes :

- Information diffusée à tous les membres de l'AFEMS et aux associations de gynécologues et obstétriciens, de sages-femmes, à la Maison Rose, au CEGID.

- Vérification des critères d'éligibilité. 
- Rencontre de la femme avec le médecin-contact pour expliquer l'étude et voir intérêt à participer. Si la femme accepte de participer :

- Le médecin informe la coordination qui organise une rencontre entre la femme et une intervieweuse formée.

- En présence d'une femme consentante, l'intervieweuse commence par expliquer l'étude de nouveau et demander le consentement éclairé.

- Difficultés rencontrées

- Refus de participer (ne veut plus se remémorer l'histoire)

- Problème de confiance de la part de la victime (médecin traitant différent de celui/celle qui fait l'interview)

\section{Présentation des résultats de l'étude}

Les résultats de l'étude ont été présentés par la chargée de programmes du Population Council. Elle a commencé par un résumé du bilan de la collecte pour tous groupes confondus et qui fait état de 15 entretiens effectivement complétés, dont 14 cas de viol et 1 cas d'inceste, sur un total de 36 candidates potentielles identifiées. Après avoir donné des informations sur les caractéristiques sociodémographiques des femmes pour chaque groupe, la présentatrice s'est focalisée sur les thèmes suivant :

\section{Expérience du viol/inceste}

Pour la majorité des femmes ayant subi un viol, il a été accompagné de violence physique. Pour les autres, il a été commis après avoir été droguées.

Le crime est souvent commis par une personne connue. Pour beaucoup de femme, c'est le silence autour du drame et la dissimulation des preuves (honte, peur de la réaction des parents, peur de la menace des agresseurs). En cas de révélation, peu de femmes bénéficient de soutien/assistance (soutien moral, accompagnement...) de l'entourage. II y a même répression dans certains cas.

\section{Expérience de la grossesse et de ses conséquences}

\section{Groupe 1 : Grossesse gardée}

Expérience de la grossesse : Les résultats montrent que ces femmes ne voulaient pas réellement garder l'enfant issu du viol/inceste, mais qu'elles se sont résignées à le faire pour des raisons telles que croyances religieuses, fatalité, peur de la loi, stade avancé de la grossesse au moment de sa découverte, volonté des proches, et peur des conséquences sanitaires d'un avortement.

Conséquences : Stigmatisation (elle-même, parfois sa mère ou son enfant) par la famille, les amis, les voisins ; Discrimination par la famille, les amis, les voisins ; Echec scolaire ; Honte /déshonneur de la famille; Rancœur envers les hommes ; Manque d'affection vis-à-vis de l'enfant/rejet ; Précarité économique ; Difficultés à se (re)marier ; Violences physiques par membres de la famille ; Déni de paternité de l'auteur/fuite de responsabilité. 


\section{Groupe 2 : Avortement / infanticide}

Expérience de la grossesse : Les femmes de ce groupe sont généralement convaincues d'avoir très bien dissimulé leur grossesse. La majorité ne veulent pas reconnaître avoir commis un infanticide et déclarent que l'enfant était mort-né ; Celles qui admettent l'infanticide insistent que c'est seulement après l'accouchement qu'elles ont décidé de se débarrasser de l'enfant.

Raisons des infanticides : Eviter la honte ; Affolement ; Peur de la réaction du mari et de la (belle-) famille ; Peur d'être la risée de l'entourage.

Conséquences : Détention prolongée/isolation ; Stigmatisation ; Perte de revenus ; Rejet familial/abandon/ rupture familiale (notamment avec ses autres enfants) ; Remords.

\section{Groupe 3 : Conséquences sanitaires graves}

2 cas présentés :

Cas 1: A gardé la grossesse par croyances religieuses et fatalisme

Conséquences : Troubles psychologiques ; Echec scolaire ; Stigmatisation ; Discrimination ; Honte/déshonneur de la famille.

Cas 2: A fait un avortement par peur (réaction des parents, déshonneur de la famille) et par volonté de poursuivre ses études.

Conséquences : Troubles psychologiques, Echec scolaire ; Stigmatisation ; Discrimination ; Douleurs pelviennes.

\section{Actions contre l'agresseur}

Très peu d'actions sont entreprises par les victimes ou leurs familles contre les agresseurs pour plusieurs raisons: Peur de détruire les relations familiales ; Disparition de l'auteur du viol/inceste ; Peur de représailles ; Agresseur inconnu ; Peur de la stigmatisation ; Difficultés à réunir les preuves nécessaires.

\section{Perceptions des victimes sur la loi}

Près de la moitié des femmes disent connaitre l'existence d'une loi interdisant l'avortement. Toutefois elles n'en maitrisent pas le contenu. Elles jugent cette loi injuste, avec de nombreuses conséquences, surtout pour les femmes. Elles souhaitent que cette loi soit modifiée. Pour certaines, l'accès à l'avortement médicalisé constitue la solution idéale pour éviter des conséquences dramatiques

\section{Contribution des intervieweuses}

Les intervieweuses ont surtout mis l'accent sur:

- La gestion des aspects émotionnels chez les participantes et les intervieweuses elles-mêmes

- Les difficultés à canaliser les victimes par rapport à l'objectif de l'étude (particulièrement pour Groupe 2)

- La difficulté pour certaines victimes à faire le lien entre viol/inceste et les conséquences sanitaires (particulièrement pour Groupe 3) 


\section{Discussions}

De nombreuses questions et contributions ont été formulées lors des discussions.

\section{Questions, regroupées par thème}

Concernant le Groupe 2

- $\quad$ : : Du point de vue des intervieweuses, les femmes rencontrées en prison se sentent-elles victimes ou bourreaux ? Manifestent-elles un besoin d'assistance ou de la culpabilité ?

- $\mathbf{R}$ : Elles se sentent surtout victimes. Des victimes qui ont manifesté un grand besoin d'assistance.

- Q : Quelle est la fiabilité des entretiens menés en prison quand on sait que la première défense c'est le déni ?

- $\mathbf{R}$ : Pas de réponse spécifique apportée à cette question.

\section{Concernant le Groupe 3}

- $\quad$ Q : Pour l'un des cas, pourquoi parler d'échec scolaire comme conséquence alors qu'on dit que la femme est commerçante?

- $\quad \mathbf{R}$ : Au moment du crime, la victime était élève. C'est suite à cette expérience qu'elle a dû abandonner l'école. 17 ans ont passés depuis. Elle est devenue commerçante.

\section{Méthodologie}

- Q : Quels sont les critères d'éligibilité pour les 3 groupes?

- $\mathbf{R}$ : En plus des critères relatifs à la définition même de chaque groupe, les participantes devaient être majeures (avoir 18 ans ou plus) au moment de l'interview.

Q : Pourquoi le critère d'âge a été limité à 18 ans puisque le viol peut avoir été commis à un âge plus jeune?

- $\quad \mathbf{R}:$ 1) L'âge de la victime au moment du viol ne fait pas partie des critères d'éligibilité. La seule chose qui était stricte est que la femme devait être majeure au moment de l'interview. Donc, des filles violées en tant que mineures auraient pu être interviewées si elle avait atteint l'âge de 18 au moment de l'étude. 2) Du point de vue éthique, une femme majeure peut seule donner son consentement éclairé de manière libre, alors que pour une mineure, il faut obtenir l'autorisation d'un parent ou gardien en plus de la sienne. Vu la sensitivité du sujet, cela ne paraissait pas approprié.

- $\mathbf{Q}$ : Pourquoi l'étude s'est limitée à la région de Dakar?

- $\mathbf{R}$ : L'étude s'est limitée à la région de Dakar à cause du caractère cosmopolite de cette région, du fait que c'est la première fois qu'une telle étude se faisait, et également pour des raisons de coûts et de temps.

Q : Qu'est-ce qui explique les nombreux cas de refus ? Est-ce que ce n'est pas un problème d'approche ? Est-ce qu'on a insisté lors de la formation sur comment faire pour convaincre à participer?

- $\quad \mathbf{R}:$ 1) Dans le cadre de la formation des intervieweuses, un accent particulier a été mis sur les conseils pour bien mener un entretien, dont la négociation de la porte d'entrée. Cependant, il est aussi important de noter que du point de vue éthique, il n'est pas permis d'insister pour faire participer quelqu'un qui ne le veut pas. 2) Les refus n'étaient pas liés à la formation des intervieweuses ou au contenu du questionnaire, mais surtout au refus des femmes de se souvenir de cette expérience douloureuse. 
- Q : Les cas de refus étant important, est-ce que est-ce que le nombre de personne touchées est suffisant pour soutenir le plaidoyer (quelle est la représentativité/fiabilité de l'échantillon pour avoir des preuves scientifiques?)

- $\quad \mathbf{R}$ : Puisqu'il s'agit d'une étude qualitative, les questions d'échantillonnage et de représentativité ne se posent pas. La nature de cette approche ne permet pas une extrapolation. Le plus important est la diversité de l'expression. Les informations obtenues sont tout à fait valables et pertinents pour le plaidoyer.

Q : Pourquoi l'étude n'a-t-elle pas opté pour des Groupes de Discussions (GD) ?

- $\quad \mathbf{R}$ : Vu la sensibilité du sujet, les GD n'auraient pas été appropriés. L'EA était la méthode de collecte la mieux indiquée pour ce genre de recherche.

Q : Quelle a été l'utilisation faite des données issues des entretiens incomplets ?

- $\quad \mathbf{R}$ : Pour les 2 cas d'EA incomplets, il s'agissait en fait d'interviews arrêtées par l'intervieweuse lorsqu'elle s'est rendue compte que bien que la grossesse avait été non désirée, elle n'était pas le résultat d'un viol ou d'un inceste.

Q : Quel a été le dispositif mis en place pour le suivi des indemnisations ?

- $\quad \mathbf{R}$ : Seuls des frais de remboursement de transport ont été prévus pour les participantes. La prise en charge des victimes n'a pas été envisagée dans le cadre de cette étude car cela pouvait soulever un problème d'éthique qui consisterait à lier cette prise en charge avec leur participation.

Suivi psycho-social des participantes

- Q : En cas de désir éventuel de suivi psycho-social, pourquoi est-ce que la prise en charge des frais est supportée par les participantes?

- $\quad \mathbf{R}$ : Plusieurs raisons ont été données, notamment que c'est la participante qui décide si et quand elle voudrait avoir un suivi, et que l'étude a une durée limitée alors que la participante peut prendre le temps qu'il lui faut.

- $\mathbf{Q}$ : Par rapport à l'état psychologique des victimes, est-ce qu'il y a eu des tendances suicidaires ?

$\mathbf{R}$ : Sur les 15 cas rencontrés, seule une victime a fait état de tendances suicidaires.

Q : Pour des questions éthiques, il serait nécessaire de s'interroger sur qui doit mener ce genre d'entretiens ? Est-ce des personnes formées sur des techniques de collecte ou bien des professionnels en suivi psychologique?

- $\mathbf{R}$ : Une intervieweuse bien formée peut conduire les entretiens de manière adéquate, par contre pour le suivi, il peut être nécessaire d'avoir un personnel spécialisé dans la santé mentale pour permettre une bonne prise en charge des victimes. Cependant, il n'est pas indispensable que ce personnel spécialisé fasse partie de l'étude. C'est pourquoi, la liste de contact a été élaborée et remise aux participantes.

Stratégies de prise en charge des victimes

Q:

Existe-t-il une stratégie nationale pour la prévention et la prise en charge des cas de viol/d'inceste ?

- $\mathbf{R}$ : Une stratégie n'existe pas en tant que telle. Voir ce qui peut être fait.

Q : Etant donné qu'il n'existe pas de base de données des femmes victimes de viol/inceste, comment faire pour avoir ce genre d'outils au MSAS ? Enregistrement anonyme online? 
- $\mathbf{R}$ : Cette étude a mis en exergue la nécessité de la constitution d'une base de données des femmes victimes de viol ou de violences basées sur le genre. A suivre.

Questions générales

- Q : Concernant les 40 détentions provisoires pour infanticide ou avortement provoqué en 2013 (dans les statistiques de la diapo 2) est-il possible de désagréger en viol ou inceste selon le cas?

- $\quad \mathbf{R}$ : Les données disponibles au niveau de l'administration pénitentiaire ne permettent pas de dissocier les cas de viol, d'infanticide et d'avortement.

- Q : Qu'attend-t-on de l'assouplissement de la loi pour les femmes déjà en prison ? Peut-on s'attendre à des effets rétroactifs?

- $\quad \mathbf{R}$ : Non, il ne peut pas y avoir d'effet rétroactif, mais cela aura certainement des effets sur la nature et la durée de la peine.

\section{Autres contributions}

- Nécessité de s'intéresser au cas des femmes incarcérées qui ont des enfants hors de la prison.

- Etude intéressante pour la Grande Bretagne qui porte un intérêt particulier aux questions de santé de la reproduction, et vient d'ailleurs de financer 2 conférences internationales y ayant trait.

- Pour le Groupe 3, l'implication des sages-femmes aurait pu permettre de toucher des candidates potentielles supplémentaires.

- Très bons résultats qui sont exploitables, même s'il y a encore beaucoup à faire pour rendre visibles les problématiques de viol et inceste.

- Résultats intéressants qui suscitent beaucoup de pistes pour l'après étude.

- Pour ce genre d'étude, prévoir des séances de débriefing du point de vue psychologique pour les intervieweuses, qui sont aussi sujettes au burnout.

\section{Recommandations}

- Mettre l'accent sur la pertinence de l'étude qui constitue un argumentaire solide dans le cadre du plaidoyer pour l'assouplissement de la loi sur l'avortement.

- Elargir le champ d'analyse par rapport aux grossesses non désirées. Ne pas mettre le focus uniquement sur viol et inceste.

- Faire des recommandations pour inclure sur tout ce qui se passe autour des victimes, les membres de la société, et pour la prise en charge de leurs besoins

- Etendre l'étude aux autres régions

- $\quad$ Etendre l'étude aux moins de 18 ans

- Formuler des recommandations pour l'application de la loi notamment par rapport aux agresseurs car les femmes ne doivent pas être les seules à être punies.

- Voir comment la société sénégalaise définit le viol. Pourquoi les gens disent viol quand cela ne correspond techniquement pas à un viol. Voir ce qui se cache derrière. Aspect à documenter. 


\section{Prochaines étapes}

- Rédiger le rapport de la rencontre.

- Finaliser et disséminer ce rapport et celui de l'étude

- Elaborer un Project Brief résumant les points clé de l'étude. 


\section{Annexes}

\section{Agenda de la rencontre}

\begin{tabular}{|c|c|c|}
\hline « L'expérience & $\begin{array}{l}\text { Rencontre de partage de } \\
\text { emmes ayant subi une grossesse non désirée au Sén }\end{array}$ & gal: Une étude qualitative » \\
\hline & Hôtel Radisson Blu, Mercredi 20 janvie & r 2016 \\
\hline & AGENDA & \\
\hline Horaires & Activités & Responsables \\
\hline $8 \mathrm{~h} 30-9 \mathrm{~h} 00$ & $\begin{array}{l}\text { Accueil et mise en place des participants } \\
\text { Mots d'introduction }\end{array}$ & Population Council \& MSAS \\
\hline $09 \mathrm{~h} 00-09 \mathrm{~h} 30$ & Contexte, objectifs et méthodologie de l'étude & $\begin{array}{l}\text { Nafissatou Diop } \\
\text { Population Council }\end{array}$ \\
\hline 09h30 - 10h15 & Déroulement de la collecte pour chaque groupe & $\begin{array}{l}\text { Coordonnatrices des } 3 \text { organisations de la } \\
\text { société civile (OSC) membres de la } \\
\text { Taskforce et partenaires de l'étude } \\
\text { - Groupe } 1 \text { : RSJ - Mme Fatou Ndiaye } \\
\text { Turpin } \\
\text { - Groupe } 2 \text { : AJS - Mme Awa } \\
\text { Tounkara Cissé } \\
\text { - Groupe } 3 \text { : AFEMS - Dr Seynabou } \\
\text { Ba Diakhaté }\end{array}$ \\
\hline $10 h 15-10 h 30$ & Pause-café & \\
\hline $10 \mathrm{~h} 30-11 \mathrm{~h} 15$ & Présentation principaux résultats & $\begin{array}{l}\text { Fatou Bintou Mbow } \\
\text { Population Council }\end{array}$ \\
\hline $11 \mathrm{~h} 15-11 \mathrm{~h} 45$ & Contribution des intervieweuses & Intervieweuses des 3 OSCs \\
\hline $11 \mathrm{~h} 45-12 \mathrm{~h} 45$ & Discussions & \\
\hline $12 \mathrm{~h} 45-13 \mathrm{~h} 00$ & $\begin{array}{l}\text { Prochaines étapes et clôture de la rencontre } \\
\text { (Nafissatou Diop, DSRSE) }\end{array}$ & MSAS \\
\hline $13 \mathrm{~h} 00$ & Déjeuner & \\
\hline
\end{tabular}


Liste des participants

\begin{tabular}{|c|c|c|}
\hline $\mathbf{N}^{\circ}$ & INSTITUTION (+ fonction des individus, si possible) & NOM \& PRENOMS \\
\hline & \multicolumn{2}{|l|}{ Assemblée nationale et autres institutions } \\
\hline 1. & Commission Santé & Mame Mbayame Gueye Dione \\
\hline 2. & Commission Santé & Haoua Dia Thiam \\
\hline 3. & Réseau des Femmes Parlementaires & Awa Gueye \\
\hline 4. & Député & Anta Sarr \\
\hline \multirow[t]{2}{*}{5.} & Assistante & Fatimata Zahra Ba \\
\hline & \multicolumn{2}{|l|}{ MSAS } \\
\hline 6. & DSRSE/Directeur & Dr Bocar Mamadou Daff \\
\hline 7. & DSRSE/SRAJ & Maguette Dia \\
\hline 8. & DSRSE/CPSRSE & Sagar Gassama \\
\hline 9. & DSRSE & Coumba Dieye \\
\hline \multirow[t]{2}{*}{10.} & DSRSE & Diouma Diouf Ndiaye \\
\hline & \multicolumn{2}{|l|}{ Ministère de l'Education Nationale } \\
\hline \multirow[t]{2}{*}{11.} & Direction du Contrôle Médicale Scolaire (DCMS) & Aminata Traore Seck \\
\hline & \multicolumn{2}{|l|}{ Taskforce } \\
\hline 12. & Taskforce-Coordinatrice & Amy Sakho \\
\hline 13. & Association des Femmes Médecins du Sénégal(AFEMS) & Dr Amy Fall Ndao \\
\hline 14. & AFEMS & Dr Nabou Ba Diakhaté \\
\hline 15. & AFEMS & Dr Daba Dione Ly \\
\hline 16. & Association des juristes Sénégalaises (AJS) & Awa Tounkara \\
\hline 17. & AJS & Kemmeltou Tambadou \\
\hline 18. & AJS & Binta Diagne \\
\hline 19. & AJVA & Saliou Ndiaye \\
\hline 20. & AJVA & Moustapha Fall \\
\hline 21. & ASBEF & Abdou Aziz Mandiang \\
\hline 22. & GEEP & Die Marie Faye \\
\hline 23. & IPAS & Bineta Ndir Diop \\
\hline 24. & Réseau Siggil Jigeen (RSJ) & Safiétou Diop Ndiaye \\
\hline 25. & RSJ & Fatou Ndiaye Turpin \\
\hline 26. & RSJ & Awa Diop \\
\hline \multirow[t]{2}{*}{27.} & Sokhna FM & Aminata Sall Diop \\
\hline & \multicolumn{2}{|l|}{ Partenaires au développement, ONG et Réseaux } \\
\hline 28. & Hôpital Général de Grand Yoff (HOGGY) & Dr Jeanne Diaw (sexologue) \\
\hline 29. & OMS- Bureau pays & Dr Mariétou Diop \\
\hline 30. & Réseau des femmes sénégalaises pour la promotion de la PF & Fatimata Dème \\
\hline \multirow[t]{2}{*}{31.} & Ambassade de Grande Bretagne & Ibrahima Bob \\
\hline & \multicolumn{2}{|l|}{ Media } \\
\hline 32. & SEN TV & Babacar Mar \\
\hline 33. & SEN TV & Awa Mbodji \\
\hline
\end{tabular}




\begin{tabular}{|r|l|l|}
\hline 34. & SUD-FM & Denisia Sambou \\
\hline 35. & Soxna FM & Absa Amar \\
\hline 36. & Direct Info & Idissa Niassy \\
\hline 37. & lelendemain.com & Babacar Ndiaye \\
\hline 38. & Apaneus & Mamadou Dieng \\
\hline 39. & Apaneus & Edouard Touré \\
\hline 40. & RDV & Rassoul Seck \\
\hline 41. & RDV & Loume Tamba \\
\hline 42. & RDV & Mame Awa Diakhaté \\
\hline 43. & Afia FM & Yvette D. Mendy \\
\hline & Population Council & \multicolumn{2}{|l|}{} \\
\hline 44. & Country Director & Nafissatou Diop \\
\hline 45. & Chargée de programmes & Fatou Bintou Mbow \\
\hline 46. & Chargé de programmes & Babacar Mané \\
\hline 47. & Office Manager & Marthe Bruce \\
\hline
\end{tabular}




\section{http://stepup.popcouncil.org}

Le STEP UP (Strengthening Evidence for Programming on Unintended Pregnancy) Research Programme Consortium œuvre en faveur d'une recherche pertinente à l'élaboration des politiques afin de promouvoir une approche basée sur des données pour améliorer l'accès à la planification familiale et à l'avortement sans risques. Nous travaillons au Bangladesh, dans la partie Nord de I'Inde, au Ghana, au Kenya et au Sénégal.

STEP UP STEP UP est coordonné par le Population Council en partenariat avec African Population and Health Research Center; icddr,b; London School of Hygiene and Tropical Medicine; Marie Stopes International; et Partners in Population and Development. STEP UP est financé par UKaid du Gouvernement du Royaume Uni. 